\title{
Impact of American Joint Committee on Cancer Eighth Edition clinical stage and smoking history on oncologic outcomes in human papillomavirus-associated oropharyngeal squamous cell carcinoma
}

\author{
Peter G. Hawkins MD, PhD ${ }^{1}$ (i) | Michelle L. Mierzwa MD ${ }^{\mathbf{1}} \quad$ | Emily Bellile MS $^{\mathbf{2}}$ | \\ William C. Jackson MD ${ }^{1}$ ｜ Kelly M. Malloy MD $^{3}$ | Steven B. Chinn MD $^{3}$ |

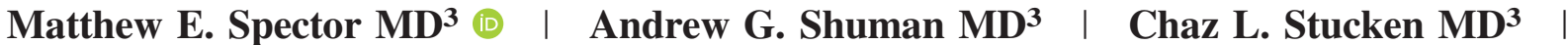 \\ Scott A. McLean MD MD $^{3}$ Carol R. Bradford MD, MS ${ }^{3}$ | Mark E. Prince MD ${ }^{3}$ | \\ Thomas E. Carey PhD Ph, $^{3,4}$ Francis P. Worden MD I $^{5}$ Paul L. Swiecicki MD \\ Jeremy M. G. Taylor PhD ${ }^{2} \quad$ Gregory T. Wolf MD $^{3}$ ｜ Avraham Eisbruch MD ${ }^{1}$ \\ Keith A. Casper $\mathrm{MD}^{3}$
}

\footnotetext{
${ }^{1}$ Department of Radiation Oncology, University of Michigan, Ann Arbor,

Email: keithcas@med.umich.edu
}

\section{Funding information}

This work was supported in part by the Newman Family Professorship Fund and RO1 CA184153 (A.E.)

\begin{abstract}
Background: The purpose of this study was to evaluate the AJCC eighth edition clinical staging system for human papillomavirus (HPV)-associated oropharyngeal squamous cell carcinoma and to further understand how clinical stage and smoking history affect oncologic outcomes. The purpose of this study was to present the understanding of how clinical stage and smoking history affect oncologic outcomes in human papillomavirus (HPV)-associated oropharyngeal squamous cell carcinoma (SCC) is critical for selecting patients for treatment deintensification.
\end{abstract}

Methods: Kaplan-Meier and Cox regression were used to evaluate overall survival (OS), locoregional recurrence-free survival (LRFS), and distant recurrence-free survival (DRFS). Concordance statistics (C-indices) were used to compare discriminating ability.

Results: The OS and DRFS but not LRFS were significantly distributed using the American Joint Committee on Cancer (AJCC) seventh and eighth editions criteria. The C-indices for OS, LRFS, and DRFS were 0.57, 0.54, and 0.60, respectively, using the AJCC seventh edition, and $0.63,0.53$, and 0.65 , respectively, using the AJCC eighth edition. On multivariate analysis, $1+$ pack-year smoking history correlated with OS (hazard ratio [HR] 1.96; 95\% confidence interval [CI] 1.2-3.1; $P<.01$ ) but not LRFS or DRFS.

Conclusion: These results support implementation of the AJCC eighth edition for HPV-associated oropharyngeal SCC. Clinical stage may be more important than smoking history in selection for deintensification.

\section{K E Y W O R D S}

American Joint Committee on Cancer, clinical staging, human papillomavirus (HPV), oropharyngeal squamous cell carcinoma, smoking 


\section{1 | INTRODUCTION}

Although oropharyngeal squamous cell carcinoma (SCC) was historically associated with tobacco smoking and alcohol consumption, the incidence of human papillomavirus (HPV)associated oropharyngeal SCC is increasing. ${ }^{1}$ Compared to smoking-related oropharyngeal SCC, HPV-positivity is associated with distinct clinical behavior, including improved prognosis. $^{2,3}$ Therefore, it was recognized that previous clinical staging systems, including the seventh edition of the American Joint Committee on Cancer (AJCC) Staging Manual, were less applicable to HPV-associated oropharyngeal SCC. ${ }^{4-6}$ In particular, patients with HPV-positive oropharyngeal SCC were observed to distribute nonuniformly among AJCC seventh edition clinical stages, which reduced prognostic utility of the system. ${ }^{5,7}$ With the goal to improve risk stratification and outcome prediction in HPV-associated oropharyngeal SCC, the International Collaboration on Oropharyngeal Cancer Network for Staging (ICON-S) developed a novel clinical staging system. This system was initially developed through a retrospective study of patients treated at Princess Margaret Hospital ${ }^{8}$ and was subsequently refined and validated in a larger, multi-institutional cohort. ${ }^{9}$ The ICON-S clinical staging system has been adapted for widespread implementation as part of the AJCC eighth edition staging manual. ${ }^{10}$ The ICON-S and AJCC eighth edition staging systems have been externally validated in 2 studies with cohorts of 150 and 279 patients each. ${ }^{11,12}$ As staging validation studies, these analyses appropriately investigated prognostication of overall survival (OS) only, and not disease-specific outcomes.

The negative effect of smoking on OS in patients with HPV-related oropharyngeal SCC is well-recognized. ${ }^{9}$ However, it is not clear if this decreased OS is related directly to differences in cancer-specific outcomes, as suggested by some studies, ${ }^{13-15}$ or merely the result of smoking-related comorbidities, as supported by others. ${ }^{16,17}$ In the ICON-S report and the related preceding study, a 20 pack-year smoking history ${ }^{8}$ and pack-years as a continuous variable ${ }^{9}$ were associated with worse OS, although potential associations with diseasespecific outcomes were not investigated. Importantly, smoking status was not incorporated into the AJCC eighth edition. ${ }^{10}$

Due to the relatively good prognosis associated with HPV-positivity in oropharyngeal SCC, efforts to deintensify treatment in these patients have been proposed and are currently under clinical investigation. Appropriate selection of candidates for deintensification relies on accurate and individualized risk stratification that considers not only OS, but disease-specific outcomes as well. We sought to describe the AJCC eighth edition staging system's ability to predict multiple cancer-specific outcomes and to investigate the impact of smoking history in a robust cohort of patients with HPVassociated oropharyngeal SCC.

\section{2 | MATERIALS AND METHODS}

\subsection{Patient population}

Patients analyzed in this institutional review board-approved study were seen at a single academic tertiary cancer center. Inclusion criteria stipulated adults with biopsy-proven oropharyngeal SCC, AJCC seventh edition stages I to IVb, positive for $\mathrm{p} 16$ by immunohistochemical staining, or, in cases in which p16 staining was not performed, HPV DNA by polymerase chain reaction. Patients were treated definitively with surgery and/or radiotherapy (RT), with or without systemic therapy (chemotherapy or cetuximab) per institutional practices, which were consistent with the National Comprehensive Cancer Network guidelines. Demographic, disease, and treatment data were collected prospectively in a passwordprotected epidemiology database. Patients provided informed consent to be included in this database. Smoking history was collected prospectively, with former smoking status defined as abstinence from tobacco use for at least 1 year before diagnosis. Patients who had quit smoking $<1$ year before diagnosis were classified as current-smokers. Patients were retrospectively restaged per the AJCC eighth edition guidelines.

\subsection{Outcome definitions and statistical methods}

Overall survival was calculated from the date of diagnosis. Patients alive at last follow-up were censored at that date. Locoregional recurrence-free survival (LRFS) and distant recurrence-free survival (DRFS) were similarly calculated from the date of diagnosis, with patients alive without evidence of locoregional or distant recurrence, respectively, being censored at the date of last follow-up. Kaplan-Meier was used to compare estimated rates of OS, LRFS, and DRFS. The log-rank test was used to evaluate the significance of outcome distribution. Concordance statistics (C-indices) were calculated to compare the discriminating ability of each staging system. Multivariate Cox regression, accounting for the AJCC eighth edition clinical group stage, was used to correlate age and smoking status with OS, LRFS, and DRFS. Age was considered as a continuous variable and smoking history was analyzed using multiple cutoffs, as detailed below.

\section{3 | RESULTS}

\section{1 | Patient characteristics and staging}

Five hundred thirty-one patients treated between 2003 and 2016 were identified and included in this analysis. Median follow-up was 48 months. Patient characteristics are shown in Table 1. Upon restaging from the AJCC seventh edition to 
TABLE 1 Patient characteristics

\section{Characteristic}

No. of patients

Age, years

Mean, years (SD)

57.9 (9.1)

Median (range)

57.1 (33-91)

Female sex $(\%)$

$69(13.0)$

Smoking status at time of diagnosis (\%)

Never

$214(40.3)$

Former

184 (34.6)

Current

$133(25.0)$

Mean pack-years (SD)

$16.2(23.2)$

$\begin{array}{ll}\text { p16 status }(\%) & \\ \text { Positive } & 316(59.5) \\ \text { Unavailable } & 215(40.5)\end{array}$

HPV DNA status (\%)

Positive

497 (93.6)

Negative

13 (2.4)

Unavailable

$21(4.0)$

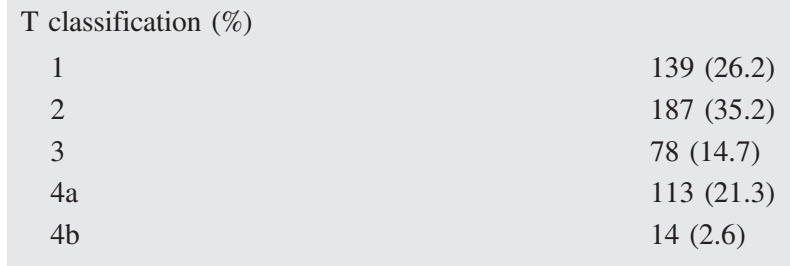

AJCC seventh edition $\mathrm{N}$ classification (\%)

$\begin{array}{ll}0 & 46(8.7) \\ 1 & 49(9.2) \\ 2 \mathrm{a} & 70(13.2) \\ 2 \mathrm{~b} & 238(44.8) \\ 2 \mathrm{c} & 90(16.9) \\ 3 & 38(7.2)\end{array}$

$\begin{array}{ll}\text { AJCC seventh edition group stage }(\%) & \\ \text { I } & 5(0.9) \\ \text { II } & 13(2.4) \\ \text { III } & 53(10.0) \\ \text { IVa } & 414(78.0) \\ \text { IVb } & 46(8.7)\end{array}$

AJCC eighth edition $\mathrm{N}$ classification (\%)

$\begin{array}{ll}0 & 46(8.7) \\ 1 & 357(67.2) \\ 2 & 90(16.9) \\ 3 & 38(7.2)\end{array}$

AJCC eighth edition group stage (\%)

I

269 (50.6)

II

108 (20.3)

III
TABLE 1 (Continued)

\begin{tabular}{|c|c|}
\hline Characteristic & No. of patients \\
\hline \multicolumn{2}{|l|}{ Treatment modality $^{\mathrm{a}}(\%)$} \\
\hline RT alone & $14(2.6)$ \\
\hline Concurrent RT + systemic therapy ${ }^{\mathrm{b}}$ & $473(89.1)$ \\
\hline Surgery followed by concurrent & $21(3.9)$ \\
\hline \multicolumn{2}{|l|}{$\mathrm{RT}+$ systemic therapy } \\
\hline Surgery followed by RT & $10(1.9)$ \\
\hline Surgery alone & $13(2.4)$ \\
\hline \multicolumn{2}{|l|}{ Treatment location (\%) } \\
\hline University of Michigan only & $462(87.0)$ \\
\hline Portion at outside facility & $69(13.0)$ \\
\hline \multicolumn{2}{|c|}{$\begin{array}{l}\text { Treatment modality refers to initial, definitive treatment, excluding diagnostic } \\
\text { procedures, such as diagnostic tonsillectomy and excisional lymph node } \\
\text { biopsy, as well as subsequent salvage and palliative therapies. }\end{array}$} \\
\hline
\end{tabular}

the eighth edition, all but 5 patients $(0.9 \%)$ were assigned a new clinical group stage, with 13 patients $(2.4 \%)$ changing from stage II to I, 25 patients (4.7\%) from stage III to I, 28 patients (5.3\%) from stage III to II, 224 patients (42.2\%) from stage IVa to I, 80 patients (15.1\%) from stage IVa to II, 110 patients $(20.7 \%)$ from stage IVa to III, and 46 patients (8.7\%) from stage IVb to III. No patient was assigned a higher clinical group stage upon reclassification.

\section{2 | Prognostication of overall survival, locoregional recurrence-free survival, and distant recurrence-free survival}

Kaplan-Meier estimates of OS by the AJCC seventh and eighth editions of clinical stage are shown in Figure 1. The log-rank test showed significant distribution of OS by the AJCC seventh and eighth editions of stages. Kaplan-Meier estimates of LRFS and DRFS are shown in Figure 2. Prognostication of DRFS was significant by use of the AJCC seventh and eighth editions of staging, although neither system yielded significant distribution of LRFS. Actuarial rates of 5year OS, LRFS, and DRFS are shown in Supporting Information Table S1. Table 2 shows hazard ratios (HRs) corresponding to the AJCC eighth edition stages for each outcome. We also attempted to calculate HRs for the AJCC seventh edition staging. Due to low numbers of patients and events, stages I and II were combined to create a reference category for OS. This approach yielded HRs of 0.50 (95\% confidence interval [CI] 0.1-2.6), 0.98 (95\% CI 0.2-4.0), and 2.21 (95\% CI 0.5-9.6) for the AJCC seventh edition stages III, IVa, and IVb, respectively. However, there were too few recurrences in stages I and II to allow for estimation of HRs for LRFS and DRFS. 

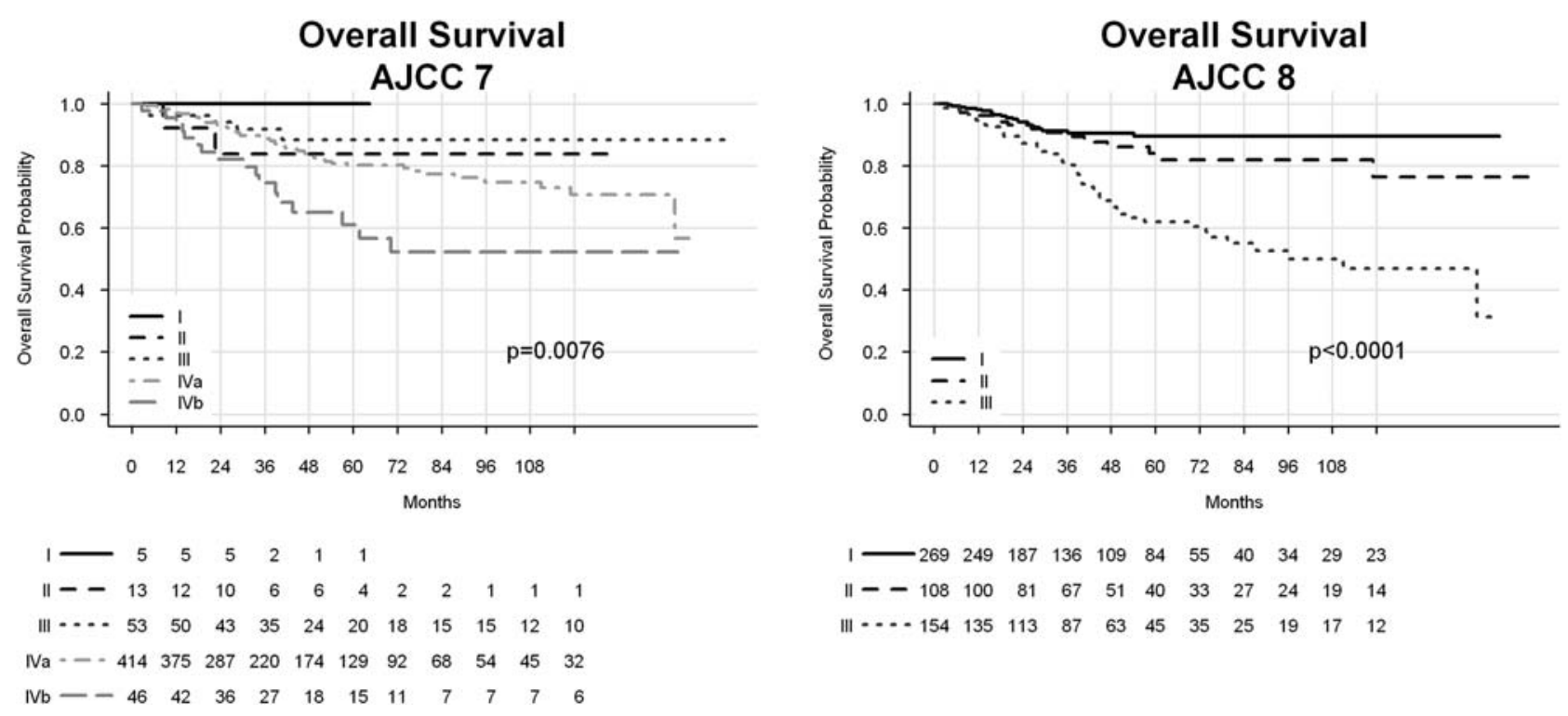

F I G U RE 1 Kaplan-Meier estimates of overall survival by the American Joint Committee on Cancer (AJCC) seventh edition and eight edition cancer staging manuals.

As both the AJCC seventh edition and eighth edition guidelines resulted in statistically significant distribution of OS and DRFS, we sought to compare the discriminating ability of these 2 systems by calculating $\mathrm{C}$-indices. Table 3 shows these results, with the AJCC eighth edition yielding higher (better) C-indices for OS and DRFS compared to the AJCC seventh edition.

\section{3 | Impact of smoking history}

In this cohort, there were no significant differences in the relative prevalence of the AJCC eighth edition T classification, $\mathrm{N}$ classification, and group stages among never smokers, current smokers, and former smokers (Supporting Information Table S2). On multivariate analysis, accounting for clinical group stage (per the AJCC eighth edition), age and any smoking history $(1+$ pack-years $)$ were statistically significantly correlated with OS, whereas smoking history by other cutoffs, namely $10+$ pack-years, $20+$ pack-years, and current smoking status, were not (Table 4). No degree of smoking history was significantly associated with LRFS or DRFS. Only age was significantly correlated with LRFS.

\section{4 | DISCUSSION}

These data confirm the improved prognostication of OS in patients with HPV-associated oropharyngeal SCC using the AJCC eighth edition clinical staging compared to the AJCC seventh edition. Although both systems yielded significant distribution of OS, the C-index associated with the AJCC eighth edition was higher than that associated with the seventh edition, indicating superior performance. This is consistent with findings from the initial ICON-S studies ${ }^{8,9}$ as well as previously published validation reports. ${ }^{11,12}$ Compared with previous validation studies, this work analyzed a larger cohort of prospectively collected patients and investigated cancer-specific outcomes in addition to OS. Although the primary goal of staging is prognostication of OS, understanding how the stage predicts locoregional and distant control can guide clinical decision making. In this regard, we found that the AJCC eighth edition clinical staging manual showed superior prognostication of DRFS compared to the seventh edition; however, neither system resulted in significant LRFS distribution. This observation is consistent with the understanding that in HPV-associated oropharyngeal SCC, distant failure is a more important cause of cancer mortality than locoregional failure, likely due to the generally excellent locoregional control achieved in these patients. ${ }^{2,18-20}$ Interestingly, the rates of distant recurrence in patients with AJCC eighth edition stages I and II were relatively similar, whereas patients with stage III disease demonstrated a much higher risk.

A major criticism of the AJCC seventh edition staging for HPV-associated oropharyngeal SCC is that patients demonstrate uneven stage distribution. ${ }^{5-7}$ Specifically, a disproportionately high number of patients are diagnosed with higher stage disease, which results in poor differentiation of outcomes. In this analysis, distribution of patients by clinical stage was markedly different using the AJCC seventh edition versus the AJCC eighth edition guidelines. We found that most patients in our cohort were initially diagnosed with stage IV disease using the AJCC seventh edition criteria, but when retrospectively reassessed using the AJCC eighth edition, a majority were assigned an early stage. This redistribution was associated with improved stratification of OS and DRFS. 

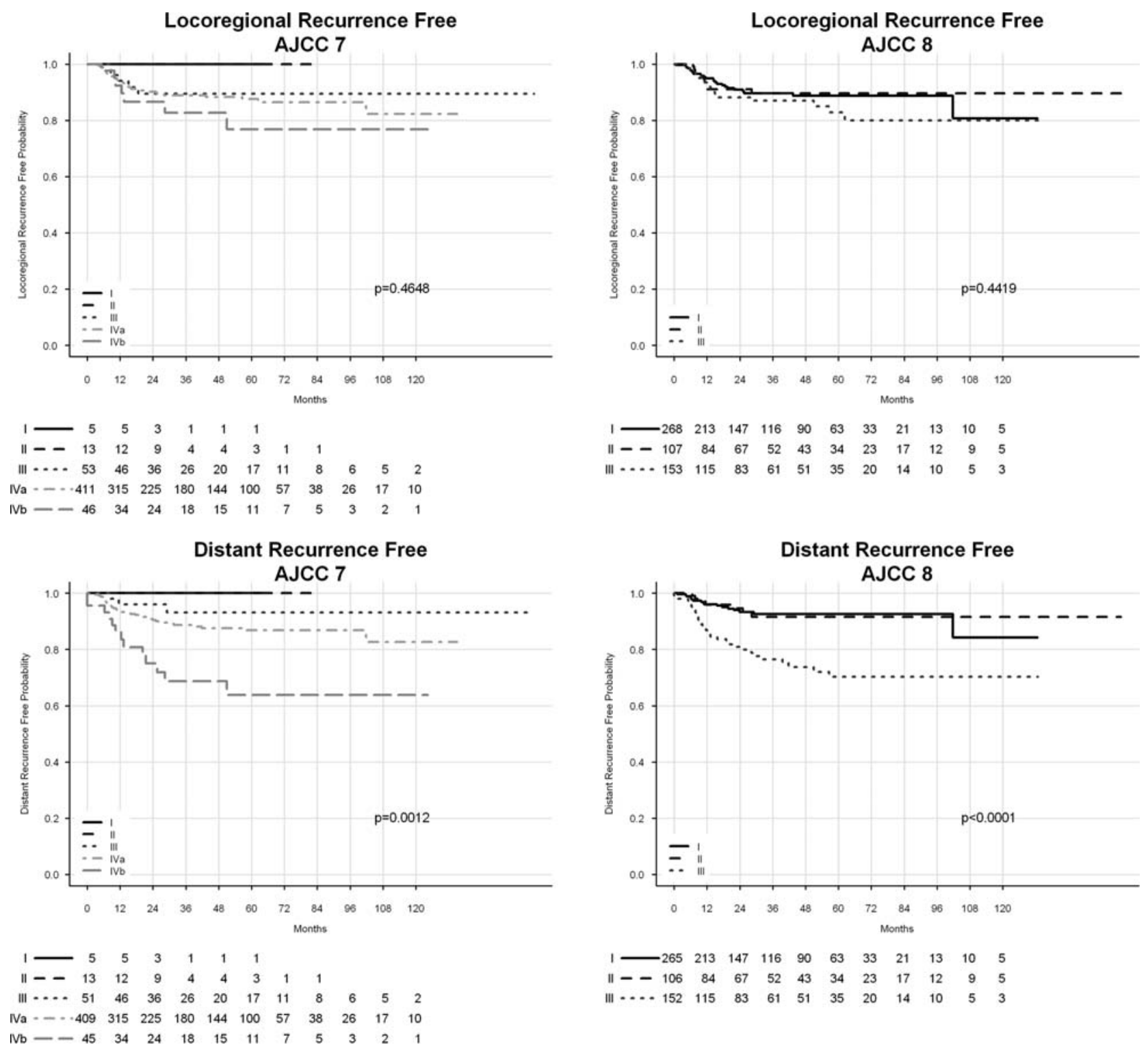

F I G URE 2 A, B, Kaplan-Meier estimates of locoregional and C, D, distant recurrence-free survival by the American Joint Committee on Cancer (AJCC) seventh edition and eighth edition cancer staging manuals.

Since the earliest studies describing HPV-associated oropharyngeal SCC, smoking has been consistently associated with worse $\mathrm{OS}^{2}$ Of particular relevance to our present work are studies that included redefined staging for HPV-associated disease. In the staging reclassification work preceding the ICON-S study, Huang et $\mathrm{al}^{8}$ identified a 20 pack-year smoking history as being associated with worse OS, particularly in patients with early-stage disease. In the subsequent ICON-S report, O'Sullivan et $\mathrm{al}^{9}$ identified smoking pack-years as a significant correlate with OS on multivariate analysis. However, neither of the 2 published validation studies found smoking to be independently correlated with OS when using updated staging. ${ }^{11,12}$ Results from the present study demonstrate a statistically significant association between OS and a $1+$ pack-year smoking history but no significant correlation using other cutoffs, including 20 pack-years.
Although the impact of smoking on OS is relatively wellestablished, it remains uncertain if this is related to mortality from noncancer smoking-related health effects or is directly related to oropharyngeal SCC outcomes. Certain studies have shown higher rates of treatment failure or disease progression in HPV-positive smokers than nonsmokers, ${ }^{13-15}$ although others have shown no impact of smoking history on diseasespecific outcomes. ${ }^{16,17}$ In the work presented here, we found that when accounting for stage and age, neither current smokers nor former smokers were at increased risk for locoregional or distant recurrence when compared with nonsmokers. These findings suggest that in patients with HPV-positive disease, smoking may impact OS primarily through mechanisms not directly related to disease recurrence.

In the era of treatment deintensification for HPV-associated oropharyngeal SCC, the identification of truly "low-risk" 
TABLE 2 Kaplan-Meier estimated hazard ratio of outcomes by American Joint Committee on Cancer seventh edition and eighth edition clinical stage manuals

\section{AJCC seventh and} eighth editions

\section{OS, HR (95\% CI)}

$\begin{array}{ll}\text { Stage I } & \text { Reference } \\ \text { Stage II } & 1.54(0.8-3.0) \\ \text { Stage III } & 4.07(2.5-6.8)\end{array}$

LRFS, HR (95\% CI)

Stage I

Reference

Stage II

$0.95(0.5-2.0)$

Stage III

$1.41(0.8-2.6)$

DRFS, HR (95\% CI)

$\begin{array}{ll}\text { Stage I } & \text { Reference } \\ \text { Stage II } & 0.97(0.4-2.3) \\ \text { Stage III } & 3.70(2.1-6.6)\end{array}$

Abbreviations: AJCC, American Joint Committee on Cancer; CI, confidence interval; DRFS, distant recurrence-free survival; HR, hazard ratio; LRFS, locoregional recurrence-free survival; OS, overall survival.

TA B LE 3 Concordance statistics calculated for overall survival, locoregional recurrence-free survival, and distant recurrence-free survival using the American Joint Committee on Cancer seventh edition versus the eighth edition clinical staging criteria

\begin{tabular}{lll} 
& AJCC seventh edition & AJCC eighth edition \\
\hline OS & 0.57 & 0.63 \\
\hline LRFS & 0.54 & 0.53 \\
\hline DRFS & 0.60 & 0.65 \\
\hline
\end{tabular}

Abbreviations: AJCC, American Joint Committee on Cancer; DRFS, distant recurrence-free survival; LRFS, locoregional recurrence-free survival; OS, overall survival.

Higher concordance statistic values indicate improved discriminating ability. patients who may be eligible for such strategies is critical. The most important factors considered in reported and ongoing deintensification trials have been stage and smoking history. In this setting, the impact of these variables on disease-specific outcomes, and not solely on OS, is particularly relevant. In the Eastern Cooperative Oncology Group study 1308, a trial of reduceddose RT and cetuximab in patients with complete clinical response to induction chemotherapy, patients with nodal classification $<\mathrm{N} 2 \mathrm{c}(<\mathrm{N} 2$ by the AJCC eighth edition criteria), T classification $<\mathrm{T} 4$, and $\mathrm{a} \leq 10$ pack-year smoking history showed substantially better OS and progression-free survival than patients with more advanced disease or a more significant smoking history. ${ }^{21}$ Some have interpreted these results to indicate that an extensive smoking history should exclude patients from deintensified treatment. Conversely, in an analysis of patients treated for HPV-associated oropharyngeal SCC with RT alone or concurrent chemoradiotherapy, O'Sullivan et al $^{16}$ found that for patients with a $>10$ pack-year smoking history, only OS, and not cancer-specific outcomes, was decreased in patients treated with RT alone compared to chemoradiotherapy. Some have suggested that these results indicate that smoking history should not disqualify patients from deintensification. After these results, some subsequent studies have excluded patients with a significant smoking history, including the Trans-Tasman Radiation Oncology Group 12.01 and the National Research Group Oncology HN002, whereas others have not, such as the Radiation Therapy Oncology Group 1016 and the Eastern Cooperative Oncology Group 3311 (reviewed in Ref. 22). This lack of consistency regarding smoking history as an exclusion criterion is not surprising given the conflicting nature of the available data. Our work indicates that smoking history may be less important than stage in relation to disease-specific outcomes, suggesting that treatment deintensification may be appropriate for otherwise low-risk patients with a significant smoking history.

There are important limitations of this study that deserve mention. Although patients were accrued prospectively, abstraction of certain data was conducted retrospectively. This raises issues of bias as well as data integrity and accuracy, in

TA B LE 4 Multivariate analysis, accounting for group stage (per American Joint Committee on Cancer eighth edition), correlating smoking history and age with overall survival, locoregional recurrence-free survival, and distant recurrence-free survival

\begin{tabular}{|c|c|c|c|c|c|c|}
\hline & $\begin{array}{l}\text { OS } \\
\text { HR }(95 \% \text { CI })\end{array}$ & $P$ value & $\begin{array}{l}\text { LRFS } \\
\text { HR }(95 \% \text { CI })\end{array}$ & $P$ value & $\begin{array}{l}\text { DRFS } \\
\text { HR }(95 \% \text { CI })\end{array}$ & $P$ value \\
\hline Any smoking & $1.96(1.2-3.1)$ & $<.01$ & $1.23(0.7-2.2)$ & .42 & $1.32(0.8-2.3)$ & .33 \\
\hline $10+$ pack-years & $1.49(1.0-2.3)$ & .05 & $1.21(0.7-2.1)$ & .41 & $1.03(0.6-1.7)$ & .95 \\
\hline $20+$ pack-years & $1.26(0.8-2.0)$ & .38 & $1.06(0.6-1.9)$ & .93 & $1.02(0.6-1.8)$ & .98 \\
\hline Age (per year increase) & $1.04(1.0-1.1)$ & $<.01$ & $1.03(1.0-1.1)$ & .03 & $1.02(1.0-1.1)$ & .14 \\
\hline
\end{tabular}

Abbreviations: CI, confidence interval; DRFS, distant recurrence-free survival; HR, hazard ratio; LRFS, locoregional recurrence-free survival; OS, overall survival. Each row represents a separate Cox model. 
addition to concerns regarding patients who were lost to follow-up. More robust prospective data that uses the AJCC eighth edition a priori is needed to more definitively address questions of selection criteria for treatment deintensification. Statistical analysis was also complicated by the low number of patients and events within certain group stages. This resulted from nonuniform distribution of patients among the AJCC seventh edition clinical stages, which highlights a significant shortcoming of that system. Another limitation to this study is the inclusion of patients whose specimens were positive for HPV DNA but for whom p16 status was unavailable. The AJCC eighth edition stipulates that p16 staining should be used to determine the HPV-relatedness of oropharyngeal cancers. However, a significant number of patients in this analysis were diagnosed before p16 staining was routinely performed at our institution. Although HPV DNA detection and p16positivity are highly correlated, ${ }^{23}$ this deviation from current guidelines should be recognized. It is also important to note that this study analyzed the impact of clinical stage only, and not pathologic stage. The AJCC eighth edition has a separate pathologic staging schema, which has been a source of early controversy. However, as questions regarding clinical stage and smoking history also apply to patients treated with surgery, we elected to include these patients without focusing on their pathologic stage. As a result, of course, we cannot use these data to comment on the AJCC eighth edition surgical staging system.

\section{5 | CONCLUSIONS}

The AJCC eighth edition clinical staging system appropriately stratifies outcomes for HPV-associated oropharyngeal SCC in terms of OS and DRFS but not LRFS. Although smoking history was associated with worse OS in this cohort, it did not correlate with LRFS or DRFS. These results support implementation of the AJCC eighth edition cancer staging manual for HPV-associated oropharyngeal SCC and suggest that smoking history should not inherently exclude patients from treatment deintensification paradigms.

\section{FUNDING INFORMATION}

This research was supported by the University of Michigan's Head and Neck SPORE grant (P50 CA97248) and additional funding supported in part by the Newman Family Professorship Fund and Ro11184153 (A.E.)

\section{ORCID}

Peter G. Hawkins MD, PhD (D) http://orcid.org/0000-00031100-9388

Matthew E. Spector MD (D) http://orcid.org/0000-0001-76466075

\section{REFERENCES}

[1] Chaturvedi AK, Engels EA, Pfeiffer RM, et al. Human papillomavirus and rising oropharyngeal cancer incidence in the United States. J Clin Oncol. 2011;29(32):4294-4301.

[2] Ang KK, Harris J, Wheeler R, et al. Human papillomavirus and survival of patients with oropharyngeal cancer. $N$ Engl J Med. 2010;363(1):24-35.

[3] Fakhry C, Westra WH, Li S, et al. Improved survival of patients with human papillomavirus-positive head and neck squamous cell carcinoma in a prospective clinical trial. J Natl Cancer Inst. 2008;100(4):261-269.

[4] Edge SB, Byrd DR, Compton C, Fritz AG, Greene FL, Trotti A III, eds. AJCC Cancer Staging Manual. 7th ed. New York, NY, Springer, 2010.

[5] Ward MJ, Mellows T, Harris S, et al. Staging and treatment of oropharyngeal cancer in the human papillomavirus era. Head Neck. 2015;37(7):1002-1013.

[6] Spector ME, Gallagher KK, Bellile E, et al. Patterns of nodal metastasis and prognosis in human papillomavirus-positive oropharyngeal squamous cell carcinoma. Head Neck. 2014;36(9): 1233-1240.

[7] Dahlstrom KR, Calzada G, Hanby JD, et al. An evolution in demographics, treatment, and outcomes of oropharyngeal cancer at a major cancer center: a staging system in need of repair. Cancer. 2013;119(1):81-89.

[8] Huang SH, Xu W, Waldron J, et al. Refining American Joint Committee on Cancer/Union for International Cancer Control TNM stage and prognostic groups for human papillomavirus-related oropharyngeal carcinomas. J Clin Oncol. 2015;33(8):836-845.

[9] O’Sullivan B, Huang SH, Su J, et al. Development and validation of a staging system for HPV-related oropharyngeal cancer by the International Collaboration on Oropharyngeal cancer Network for Staging (ICON-S): a multicentre cohort study. Lancet Oncol. 2016;17(4):440-451.

[10] Amin MB, Edge SB, Greene F, eds. AJCC Cancer Staging Manual. Eighth Ed. New York, NY, Springer, 2017.

[11] Würdemann N, Wagner S, Sharma SJ, et al. Prognostic impact of AJCC/UICC 8th edition new staging rules in oropharyngeal squamous cell carcinoma. Front Oncol. 2017;7:129.

[12] Porceddu SV, Milne R, Brown E, et al. Validation of the ICON$\mathrm{S}$ staging for HPV-associated oropharyngeal carcinoma using a pre-defined treatment policy. Oral Oncol. 2017;66:81-86.

[13] Maxwell JH, Kumar B, Feng FY, et al. Tobacco use in human papillomavirus-positive advanced oropharynx cancer patients related to increased risk of distant metastases and tumor recurrence. Clin Cancer Res. 2010;16(4):1226-1235.

[14] Gillison ML, Zhang Q, Jordan R, et al. Tobacco smoking and increased risk of death and progression for patients with p16positive and p16-negative oropharyngeal cancer. J Clin Oncol. 2012;30(17):2102-2111.

[15] Vainshtein JM, Spector ME, McHugh JB, et al. Refining risk stratification for locoregional failure after chemoradiotherapy in human papillomavirus-associated oropharyngeal cancer. Oral Oncol. 2014;50(5):513-519.

[16] O’Sullivan B, Huang SH, Perez-Ordonez B, et al. Outcomes of HPV-related oropharyngeal cancer patients treated by radiotherapy 
alone using altered fractionation. Radiother Oncol. 2012;103(1): 49-56.

[17] O'Sullivan B, Huang SH, Siu LL, et al. Deintensification candidate subgroups in human papillomavirus-related oropharyngeal cancer according to minimal risk of distant metastasis. $J$ Clin Oncol. 2013;31(5):543-550.

[18] Trosman SJ, Koyfman SA, Ward MC, et al. Effect of human papillomavirus on patterns of distant metastatic failure in oropharyngeal squamous cell carcinoma treated with chemoradiotherapy. JAMA Otolaryngol Head Neck Surg. 2015;141(5): 457-462.

[19] Huang SH, Perez-Ordonez B, Weinreb I, et al. Natural course of distant metastases following radiotherapy or chemoradiotherapy in HPV-related oropharyngeal cancer. Oral Oncol. 2013;49(1): 79-85.

[20] Huang SH, Perez-Ordonez B, Liu FF, et al. Atypical clinical behavior of p16-confirmed HPV-related oropharyngeal squamous cell carcinoma treated with radical radiotherapy. Int J Radiat Oncol Biol Phys. 2012;82(1):276-283.

[21] Marur S, Li S, Cmelak AJ, et al. E1308: Phase II trial of induction chemotherapy followed by reduced-dose radiation and weekly cetuximab in patients with HPV-associated resectable squamous cell carcinoma of the oropharynx - ECOG-ACRIN Cancer Research Group. J Clin Oncol. 2017;35(5):490-497.
[22] Masterson L, Moualed D, Liu ZW, et al. De-escalation treatment protocols for human papillomavirus-associated oropharyngeal squamous cell carcinoma: a systematic review and meta-analysis of current clinical trials. Eur J Cancer. 2014;50(15):2636-2648.

[23] Reimers N, Kasper HU, Weissenborn SJ, et al. Combined analysis of HPV-DNA, p16 and EGFR expression to predict prognosis in oropharyngeal cancer. Int J Cancer. 2007;120(8): 1731-1738.

\section{SUPPORTING INFORMATION}

Additional Supporting Information may be found online in the supporting information tab for this article.

How to cite this article: Hawkins PG, Mierzwa ML, Bellile E, et al. Impact of American Joint Committee on Cancer Eighth Edition clinical stage and smoking history on oncologic outcomes in human papillomavirusassociated oropharyngeal squamous cell carcinoma. Head \& Neck. 2019;41:857-864. https://doi.org/10. 1002/hed.25336 